\title{
Uses of the Novel Small Peptide, KTH-222, in Treating Human Pancreatic Cancer: Evaluation of Different Treatment Regimens using a Mouse Xenograft Model
}

\author{
Michael R Kozlowski ${ }^{1 *}$ and Roni E Kozlowski ${ }^{2}$ \\ ${ }^{1}$ Arizona College of Optometry, Midwestern University, United States of America
}

${ }^{2}$ Kalos Therapeutics Incorporated, United States of America

*Corresponding author: Michael R. Kozlowski, Arizona College of Optometry, Midwestern University, Glendale, Arizona, United States of America

\section{ARTICLE INFO}

Received: 业 May 18, 2021

Published: 慧 May 25, 2021

Citation: Michael R Kozlowski, Roni E Kozlowski. Uses of the Novel Small Peptide, KTH-222, in Treating Human Pancreatic Cancer: Evaluation of Different Treatment Regimens using a Mouse Xenograft Model. Biomed J Sci \& Tech Res 36(1)-2021. BJSTR. MS.ID.005798.

Keywords: Pancreatic Cancer; Chemotherapy; Small Peptide; Atrial Natriuretic Peptide; KTH-222

\begin{abstract}
KTH-222 is a novel, 8-amino acid length peptide derived from atrial natriuretic peptide and a group of related peptides that reduce cancer cell growth. It was previously reported that KTH-222 reduces the rate of tumor growth and prolongs survival in mice implanted with MIA PaCa-2 human pancreatic cancer cells more effectively than gemcitabine when used as primary therapy. The goal of the present study is to explore the effectiveness of KTH-222 for treating pancreatic cancer when used in two other roles: combination primary therapy and salvage therapy. Female athymic nude mice were inoculated subcutaneously in the right flank with MIA-PaCa-2 tumor cells. KTH-222 was administered as part of a combination primary therapy together with gemcitabine and paclitaxel or as a salvage therapy. When used in combination with gemcitabine and paclitaxel as primary therapy, KTH-222 further reduced tumor volume, although it did not extend the interval until terminal tumor volume was reached. When used a salvage therapy, KTH-222 did extend the interval until terminal tumor volume was reached and, in one case, produced a sustained regression in tumor growth. The results of this study, together with earlier results, suggest that KTH-222 could be used in several different ways in the treatment of pancreatic cancer.
\end{abstract}

\section{Introduction}

There is an ongoing need for the discovery of new chemotherapies that improve the treatment of pancreatic cancer [1-5]. This disease is the third leading cause of cancer-related deaths in the United States, with a 5-year survival rate of only $4 \%$ to $7 \%$ [6]. The two major, standard-of-care treatments for advanced pancreatic cancer are gemcitabine plus protein-bound paclitaxel, and FOLFIRINOX (5-fluorouracil, leucovorin, irinotecan, and oxaliplatin) [5, 7-10]. While initially very effective, the long-term utilities these treatment regimens are ultimately limited by toxicity and the development of resistance.

A growing body of evidence supports a role for atrial natriuretic peptide (ANP) and a group of peptides related to it by sequence homology $[11,12]$ or by sharing the same precursor protein [13] in the treatment of cancer. These peptides inhibit the proliferation of human pancreatic cancer cells, as well as those from human lung, hepatic, and gastric cancers, in culture [11-19]. Certain of these peptides have also been shown to reduce the growth of tumors derived from human pancreatic cells, as well as those from human breast and lung cancer cells, in mouse xenograft models [13,19-22]. Although the effects of these peptides on treating cancer in humans has not yet been well-explored, ANP itself has been reported to reduce metastases after curative lung cancer surgery in human clinical studies [23].

The discovery of drugs for the treatment of pancreatic cancer based on the structure of ANP and related peptides has been 
difficult since peptides related to ANP through a common precursor, such kaliuretic peptide, long-acting ANP, and vessel dilator peptide, share no obvious sequence homology with ANP or with each other, even though they all inhibit cancer cell growth [13]. More careful analysis, however, reveals that all of the peptides related to ANP that reduce the growth of cancer cells contain a common, 8-amino acid length motif. The version of this motif that was optimized for activity against pancreatic cancer is the peptide, KTH-222 [19].

KTH-222 inhibits the attachment, proliferation, and development of an invasive morphology in cultured human pancreatic tumor cells [19]. The ability of KTH-222 to interfere with tubulin dynamics may be responsible for these effects. Furthermore, in mice xenografted with MIA PaCa-2 human pancreatic cancer cells, KTH-222 reduced the rate of tumor growth and prolonged survival more effectively, and with less evidence of toxicity, than gemcitabine when used was as sole primary therapy [19].

In order to advance KTH-222 into clinical development as a treatment for pancreatic cancer, it is desirable to select the optimal treatment regimen for clinical trials. This report uses a mouse xenograft model system of human pancreatic cancer to evaluate two additional potential treatment regimens for KTH-222: combination primary therapy together with the gemcitabine plus paclitaxel, and salvage therapy following treatment with gemcitabine plus paclitaxel followed by maintenance treatment with sunitinib.

\section{Materials and Methods}

\section{Materials}

KTH-222 (NH2-LKGQLRCI-CO2H) was synthesized at > 95\% purity by New England Peptides (Gardner, MA). KTH-222 was stored lyophilized at $4^{\circ} \mathrm{C}$ and dissolved in saline just prior to use. Gemcitabine was received as a colorless solution and was stored at $4^{\circ} \mathrm{C}$ until use. Prior to use, Gemcitabine was diluted to the desired concentration in a saline solution. Paclitaxel was also received as a liquid and stored at $25^{\circ} \mathrm{C}$, until formulated for use in saline, after which it was stored at $4^{\circ} \mathrm{C}$. Sunitinib was received from Lily and stored as a powder. It was dissolved in saline prior to use. MIA-PaCa-2 human pancreatic adenocarcinoma cells (CRL1420) were received from the American Type Culture Collection (ATCC; Manassas, VA) and were cultured according to the supplier's instructions.

\section{Xenograft Model System}

The xenograft studies were performed at Translational Drug Development (TD2, Scottsdale, AZ) using their standard protocol, which was approved by the TD2 institutional review board (Approved Study \#TD3651). Female athymic nude mice were received at 4 weeks of age and were acclimated for at least 5 days prior to study initiation. The mice were housed in microisolator cages and maintained under specific, pathogen-free conditions.
The mice were fed Toland Global Diet ${ }^{\circledR}$ 2920x irradiated laboratory animal diet, and autoclaved water was freely available. All procedures were carried out under the institutional guidelines of Translational Drug Development Institutional Animal Care and Use Committee, which conform to the Guide for the Care and Use of Laboratory Animals as adopted and promulgated by the U.S. National Institutes of Health (Protocol Number TD3651). Animals were identified using transponders.

The mice were inoculated subcutaneously in the right flank with $0.1 \mathrm{ml}$ of a $50 \%$ media / $50 \%$ Matrigel $\AA$ mixture containing a suspension of $5 \times 106$ MIA-PaCa- 2 tumor cells. At the time of inoculation, the mice were 5-6 weeks old. Tumor bearing animals were monitored and tumors were measured periodically until they reached a size of approximately $125 \mathrm{~mm}^{3}$. Tumor width and length diameters were measured using a digital caliper. The measured values were digitally recorded using animal study management software, Study Director. Tumor volumes were calculated utilizing the formula: Tumor volume $\left(\mathrm{mm}^{3}\right)=(\mathrm{a} \times \mathrm{b} 2 / 2)$ where ' $\mathrm{b}$ ' is the smallest diameter and ' $a$ ' is the largest diameter [24]. On the thirteenth day following inoculation, designated Study Day 1, forty mice with tumor sizes of $129-188 \mathrm{~mm}^{3}$ were randomized into three groups of ten plus two groups of five (Table I), each with a mean tumor volume of approximately $160 \mathrm{~mm}^{3}$, by random equilibration using Study Director. The drug or vehicle treatments were started on Study Day 1. Tumor volumes and body weights were recorded when the mice were randomized and were measured again twice weekly thereafter. Clinical observations were made daily. Mice in a group were humanely euthanized when the average tumor volume for the group reached or exceeded $1000 \mathrm{~mm}^{3}$. This volume, called the terminal tumor volume (TTV) was determined by experience to occur just before morbidity or mortality occurs in the group. One mouse in Group 5 who demonstrated tumor regression was kept alive after the group reached TTV.

\section{Drug Treatments}

Combination Primary Therapy: The dosing regimen for the combination primary therapy evaluation is shown at the in Table I, section A. Gemcitabine ( $80 \mathrm{mg} / \mathrm{kg}$ at $10 \mathrm{mg} / \mathrm{ml}$, intraperitoneal) and paclitaxel $(30 \mathrm{mg} / \mathrm{kg}$ in $10 \mathrm{mg} / \mathrm{kg}$ saline, intravenous) were administered together every third day for 4 cycles beginning on Study Day 1 (i.e., on Studies Days 1, 4, 7, 10) (Groups 2 and 3). In Group 3, KTH-222 was administered during and following the administration of the other drugs, 3 times per week $(0.5 \mathrm{mg} /$ $\mathrm{kg}$ at $10 \mathrm{mg} / \mathrm{kg}$, intravenously) beginning on Study Day 1 and continuing until the termination of the group. Group 1 received vehicle treatments of saline alone and were given at the same times and volumes as the drug treatments. The dose of KTH-222 was determined as previously described [19]. The doses for each of the other drugs were selected by TD2 based on their experience in using 
these drug combinations in this xenograft model and were chosen to produce the greatest anti-tumor effects without producing druginduced morbidity or mortality.

Salvage Therapy: Two groups received the same gemcitabine plus paclitaxel primary therapy as did Group 2, followed by maintenance therapy with sunitinib $(40 \mathrm{mg} / \mathrm{kg}$ at $10 \mathrm{mg} / \mathrm{kg}$, orally; Study Days 13-34; Table 1, section B). One of these groups (Group 5) also received KTH-222 treatment $(0.5 \mathrm{mg} / \mathrm{kg}$ at $10 \mathrm{mg} / \mathrm{kg}$, intravenously) beginning on study day 41, and continuing every third day until the end of the experiment. The other group (Group 4) received vehicle injections in place of the KHT-222 injections.

Statistical Comparisons: Group means were compared by means of a two-way ANOVA using the Bonferroni correction for multiple between-group comparisons (IBM SPSS $®$ Statistics v. 25, IBM, Armonk, NY). Individual means were compared using t-tests.

Table 1: Drug treatment regimens by group for combination primary treatments (A; top of table) and salvage treatment regimens (B; bottom of table). "Days" indicate Study Days.

\begin{tabular}{|c|c|c|c|c|}
\hline \multicolumn{5}{|c|}{ A. COMBINATION PRIMARY THERAPY } \\
\hline Group Number & $\mathrm{N}$ & Days 1-12 & \multicolumn{2}{c|}{ Vehs 13-End } \\
\hline 1 & 10 & Vehicle & KTH-222 \\
\hline 2 & 10 & Gemcitabine + Paclitaxel & Days 44-End \\
\hline 3 & 10 & Gemcitabine + Paclitaxel + KTH-222 & Days 13-34 \\
\hline \multicolumn{7}{|c|}{ B. SALVAGE THERAPY } & Sunitinib & Vehicle \\
\hline Group Number & $\mathrm{N}$ & Days 1-12 & Sunitinib & KTH-222 \\
\hline 4 & 5 & Gemcitabine + Paclitaxel & Gemcitabine + Paclitaxel & \\
\hline
\end{tabular}

\section{Result}

\section{Combination Primary Therapy}

The tumor volumes in both the group treated with gemcitabine plus paclitaxel alone (Group 2) and the group treated with gemcitabine plus paclitaxel and KTH-222 (group 3) were significantly less than those of the group treated with vehicle (Group 1; two-way ANOVA, $\mathrm{p}<.001$ for each drug-treated group compared to vehicle treatment; Figure 1). The tumor volumes in the group treated with gemcitabine plus paclitaxel plus KTH-222 were significantly smaller than those of the group treated with gemcitabine plus paclitaxel alone between Study Day 24 (when the standard deviations of the tumor volumes of the two groups became non-overlapping, Figure 1) and Study Day 44 (when the standard deviations resumed overlapping; two-way ANOVA, p < $.05)$. The vehicle treated group reached its terminal tumor volume (TTV) on Study Day 33. The drug-treated groups both reached TTV on Study Day 57.

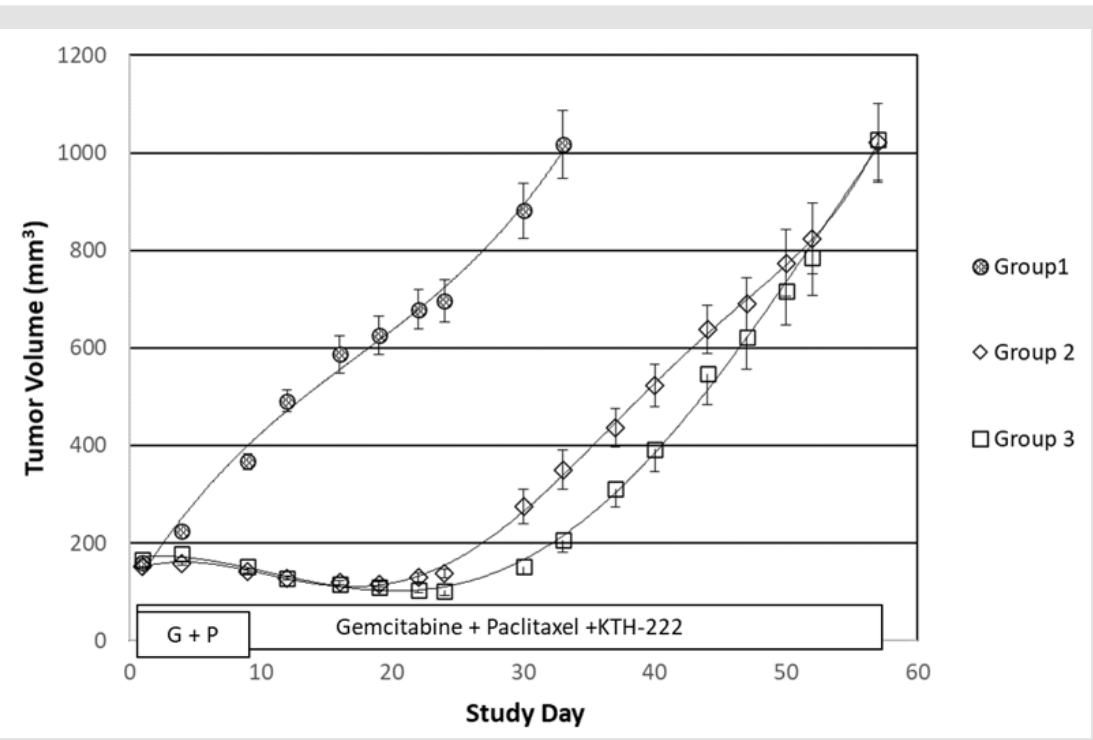

Figure 1: Combination primary therapy results. The curves show the growth of tumors from MIA PaCa-2 human pancreatic cancer cells xenografted into athymic mice. "Study Day" indicates days since initiation of treatment (see "Methods"). The treatment groups are the same as in Table IA. The administration periods for gemcitabine plus paclitaxel primary therapy (G + P; Group 2) and gemcitabine plus paclitaxel plus KTH-222 (Group 3) are indicated in boxes. Error bars indicated standard errors. 


\section{Salvage Therapy}

The tumor volumes of animals given gemcitabine plus paclitaxel primary therapy followed by sunitinib maintenance therapy appeared reduced compared to those only treated with the primary therapy (Group 2; Figure 2) but this difference did not achieve statistical significance over the entire course of the experiment
(Group 2; two-way ANOVA, $\mathrm{p}=0.053$ ). The time to TTV in Group 4 was, however, extended one week beyond that of Group 2 (Figure 2). In contrast, the tumor volumes of animals given KTH-222 salvage treatment beginning on study day 41 , following the same primary and maintenance therapies (Group 5), were significantly less than those of Group 2 (two-way ANOVA, $\mathrm{p}<0.05$ ), and the time to TTV in Group 5 was extended beyond that of Group 2 by two weeks.

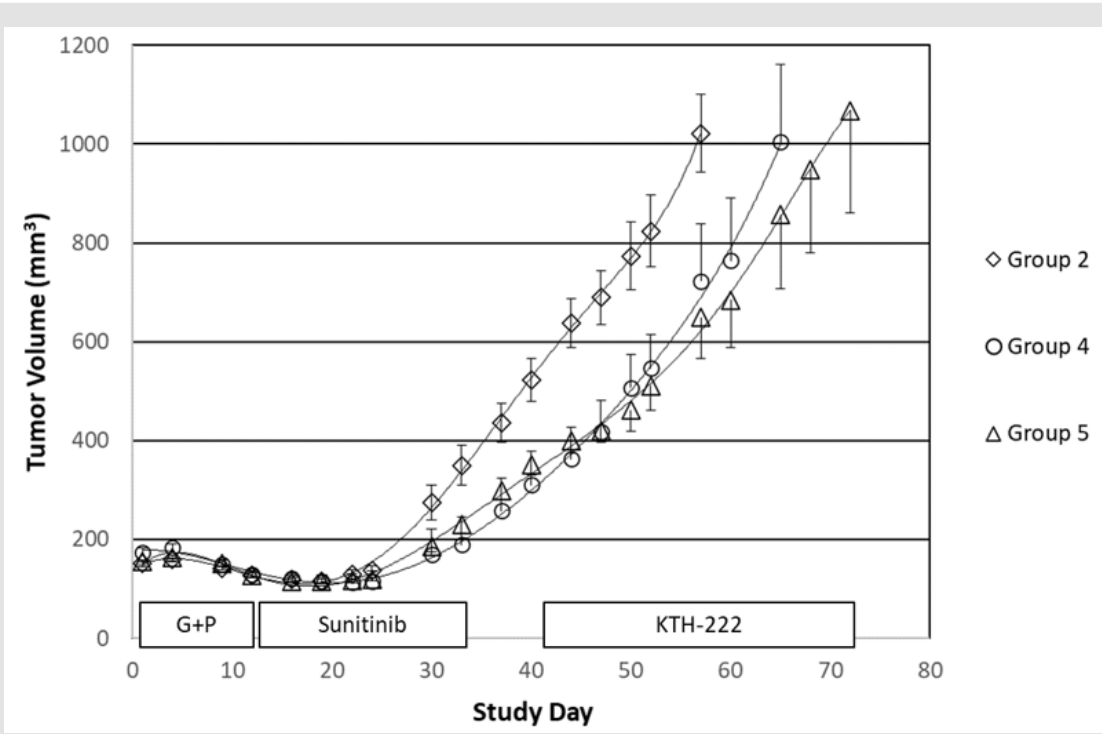

Figure 2: KTH-222 salvage therapy results. Again, the curves show the growth of tumors and "Study Day" indicates days since initiation of treatment (see "Methods"). The treatment groups are the same as in Table IB. The administration periods for gemcitabine plus paclitaxel primary therapy (G + P; Group 2) sunitinib maintenance therapy (Groups 4 and 5), and KTH-222 salvage therapy (Group 5) are indicated in boxes.

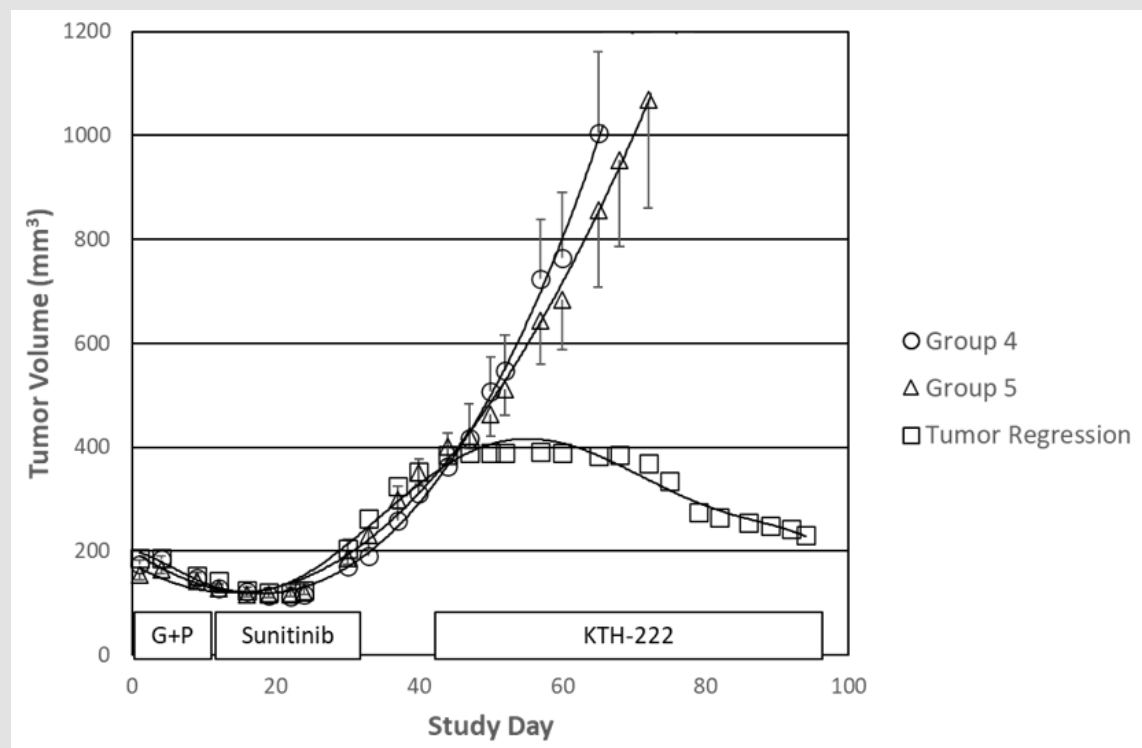

Figure 3: KTH-222-induced tumor regression. Labels and values are as in Figure 2. Tumor volumes for the animal in Group 5 with the tumor regression are indicated by squares.

Interestingly, sustained tumor regression was seen in one of the five animals in the group that received KTH-222 salvage therapy (Group 5; Figure 3). The tumor growth rate decreased to approximately $1 \%$ between Study Days 44 and 47 . The volume of the tumor began to decrease from a maximum of $392 \mathrm{~mm}^{3}$ on Study Day 60 and continued to decline through the rest of the experiment. The study was arbitrarily terminated on Study Day 94 at which time the animal with the regressing tumor was still healthy, and the 
tumor volume was continuing to decrease. By the time the study was terminated, the tumor volume in this animal had decreased by $41.1 \%$. No tumor regressions were seen in vehicle treated animals (Group 1). In addition, none of the animals in any of the other drugtreated groups showed tumor regression after Study Day 34 (final day of sunitinib maintenance therapy).

\section{Adverse Effects}

The only adverse effects seen in animals treated with KTH222 were a mild hunched posture (two animals in Group 3 and one in Group 5) and slight emaciation (1 animal in Group 3). For comparison, one animal of the control group also exhibited slight emaciation and two had hunched postures. No adverse effects were seen in the animals treated with gemcitabine plus paclitaxel alone or with sunitinib since the doses of those drugs had been chosen to avoid toxicities (see "Methods").

\section{Discussion}

KTH-222 is a novel peptide composed of 8-amino acids whose sequence is based on a motif found in ANP and a group of related peptides, all of which reduce cancer cell growth [11-17,20, 21,25]. Employing mice xenografted with human pancreatic cancer cells, we previously demonstrated that KHT-222 was more effective than gemcitabine in reducing the growth of tumors when used as the primary therapy [19]. In particular, KTH-222 produced a significantly greater reduction in tumor volume than gemcitabine in more advanced stages of tumor growth. Because KTH-222 displayed little evidence of toxicity in that study, it appeared to be good candidate for use with other anti-cancer agents in roles such as combined primary therapy and salvage therapy [19]. The present report examined the effectiveness of KTH-222 in decreasing the growth of pancreatic cancer cells in these alternative roles using the same mouse xenograft system.

In the combination primary therapy application, KTH-222 increased the effectiveness of gemcitabine plus paclitaxel therapy, a standard of care in pancreatic cancer treatment [5,7-10], in reducing tumor volume between Study Days 24 and 34. The time to TTV, however, was not extended by the addition of KTH-222 to the primary therapy. Time to terminal tumor volume can be considered an indicator of overall survival since, generally speaking, morbidity and lethality begin to occur in members of the group after this value is exceeded. The attenuation of the enhanced reduction in tumor volume by KTH-222 later in treatment period, as indicated by not extending the time to TTV, may occur because KTH-222 has biochemical actions that are somewhat opposed to those of paclitaxel. Paclitaxel promotes the assembly of microtubules and stabilizes them while KTH-222 inhibits microtubule elongation [26]. Over time, these competing actions may reduce the effectiveness of this particular drug combination. This attenuation in effectiveness of KTH-222, therefore, may not be seen in other treatment combinations that do not include taxanes.

Even if adding KTH-222 to the standard of care treatment regimen of gemcitabine and paclitaxel does not improve overall survival in human patients, as suggested by it not producing an extension of time to TTV, it could still have important clinical utility in human cancer care. For example, by reducing tumor load KTH222 might also be expected to reduce cancer-related morbidity and, thereby, improve quality of life (QOL) in human patients. Thus, it may have the same benefits as palliative cancer care $[27,28]$. Palliative care has been shown to not only increase QOL but also extend median survival in human patients, perhaps through a combination of enhancements in both function and mood [27].

To test the use of KTH-222 as salvage therapy, it was given when tumor growth had resumed after gemcitabine plus paclitaxel primary therapy and sunitinib maintenance therapies had been given. Although sunitinib is not routinely used as maintenance therapy in pancreatic cancer, it been shown to be effective in this role [29]. The tumor volumes in animals given KTH-222 salvage therapy were not significantly different than those given vehicle injections during the salvage therapy phase. This may be due to the small group sizes used. Differences between these groups that favored the KTH-222 treatment were, however, discernable. First of all, salvage treatment with KHT-222 following maintenance therapy with sunitinib significantly reduced tumor growth compared to animals who received the same primary treatment but neither the maintenance nor salvage therapies. In contrast, the tumor growth reduction in the group that received the same primary and maintenance therapies but with no salvage therapy did not achieve clinical significance with this group size. This suggests a better outcome with KTH-222 salvage therapy. Furthermore, KTH-222 salvage therapy increased the time to TTV by 14 days compared to the group given only primary therapy, and seven days compared to the group also given maintenance therapy but no KTH-222 salvage therapy.

KTH-222 salvage therapy also produced, in one of the five treated animals, a regression in tumor growth that was sustained through the remainder of the study. Tumor volume plateaued in this animal soon after the beginning of KTH-222 administration and regression was noted 19 days later. This animal was still healthy and in regression when the study was arbitrarily terminated on Study Day 94. Based on the trajectory of the tumor volume curve (Figure 3) this animal might have had a complete regression if the study had been extended. A similar effect was seen in a previous study in which KTH-222 was used as primary therapy [19]. In that study, KTH-222 essentially halted tumor growth $(<1.5 \%$ increase in volume per week) in one of the 10 animals treated. This effect was also late-occurring and persisted through the remainder of the study. None of the animals in other groups in either of these studies $(n=80)$ showed persistent tumor regression. 
The tumor regression that occurred in one of the five animals treated with KTH-222 salvage therapy could have been due to a difference in the cells comprising the tumor of the affected animal. This, however, does not seem likely since all mice were xenografted from the same culture. It also may have been due to some peculiarity in the physiology of the mouse, but this also seems improbable since all mice were of the same strain. Alternatively, in may be that small differences in injection sites placed the xenografted cells in environments which varied in how supportive they were to tumor growth. The ability of the cells to attach and invade would have been compromised by the interference in tubulin dynamics produced by KTH-222 [19], and this may have been sufficient to produce regression in less favorable environments. In addition, if the growth fraction was reduced by an inhospitable environment, the inhibitory effect of KTH-222 on cell division may also have made it impossible for the tumor to expand [19]. Such heterogeneity in growth environments also occurs with human tumors, particularly those resulting from metastases, raising the possibility that KTH222 treatment could also produce the regression of certain human tumors [30].

Taken together, the results obtained to date suggest that KTH222 has the potential to be a useful treatment for pancreatic cancer in a variety of roles. In particular, the results from the current study suggest the utility of KTH-222 in combined primary and salvage therapy treatment regimens. Those of a previous study demonstrated the effectiveness of KTH-222 as a sole primary therapy [19]. Further research be necessary to confirm the best use(s) of KTH-222 in the treatment of pancreatic cancer and, thereby, to position the peptide to enter clinical trials.

\section{Acknowledgements}

The authors gratefully acknowledge the sponsorship of this research by Kalos Therapeutics Incorporated as well as the many helpful contributions to this research by its president, Mr. George Colberg, and the Kalos Scientific Advisory Board. The authors would also like to recognize the excellent work of Paul Gonzales, Mario Sepulveda, and Anthony Lozano of TD2 in carrying out the xenograft study. Finally, the authors would like to thank Dr. Amy Stein for her consultation on the statistical analyses.

\section{Funding}

This research was funded by a sponsored research agreement between Kalos Therapeutics Incorporated and Midwestern University.

\section{Institutional Animal Care and Use Committee Statement}

All procedures were carried out under the institutional guidelines of Translational Drug Development Institutional Animal
Care and Use Committee, which conform to the Guide for the Care and Use of Laboratory Animals as adopted and promulgated by the U. S. National Institutes of Health (Protocol Number TD3651).

\section{Conflict-of-Interest Statement}

Roni E. Kozlowski is an employee, and Michael R. Kozlowski is a consultant, of Kalos Therapeutics Incorporated.

\section{References}

1. Mukhtar E, Adhami VM, Mukhtar H (2014) Targeting microtubules by natural agents for cancer therapy. Mol Cancer Ther 13: 275-284.

2. Orr GA, Verdier-Pinard P, McDaid H, Horwitz SB (2003) Mechanisms of Taxol resistance related to microtubules. Oncogene 22: 7280-7295.

3. Zhang Y, Yang SH,Guo XL (2017) New insights into Vinca alkaloids resistance mechanism and circumvention in lung cancer. Biomed Pharmacother 96: 659-666.

4. Sherrod AM, Brufsky A, Puhalla S (2011) A case of late-onset gemcitabine lung toxicity. Clin Med Insights Oncol 5: 171-176.

5. Adel N (2019) Current treatment landscape and emerging therapies for pancreatic cancer. Am J Manag Care 25: S3-S10.

6. Yabar CS, Winter JM (2016) Pancreatic Cancer: A Review. Gastroenterol Clin North Am 45: 429-445.

7. Varadhachary GR, Wolff RA (2016) Current and Evolving Therapies for Metastatic Pancreatic Cancer: Are We Stuck with Cytotoxic Chemotherapy? Journal of Oncology Practice 12: 797-805.

8. Wachtel MS, Xu KT, Zhang Y, Chiriva-Internati M, Frezza EE (2008) Pancreas cancer survival in the gemcitabine era. Clin Med Oncol 2: 405413.

9. Grossberg AJ, Chu LC, Deig CR, Fishman EK, Hwang WL, et al. (2020) Multidisciplinary standards of care and recent progress in pancreatic ductal adenocarcinoma. CA Cancer J Clin 70: 375-403.

10. Mohammad AA (2018) Advanced pancreatic cancer: The standard of care and new opportunities. Oncol Rev 12: 370 .

11. Lelievre V, Pineau N, Hu Z, Ioffe Y, Byun JY, et al. (2001) Proliferative actions of natriuretic peptides on neuroblastoma cells. Involvement of guanylyl cyclase and non-guanylyl cyclase pathways. J Biol Chem 276: 43668-43676.

12. Rashed HM, Sun H, Patel TB (1993) Atrial natriuretic peptide inhibits growth of hepatoblastoma (HEP G2) cells by means of activation of clearance receptors. Hepatology 17: 677-684.

13. Vesely DL (2013) Cardiac hormones for the treatment of cancer. Endocr Relat Cancer 20: R113-125.

14. Ohsaki Y, Yang HK, Le PT, Jensen RT, Johnson BE (1993) Human small cell lung cancer cell lines express functional atrial natriuretic peptide receptors. Cancer Res 53: 3165-3171.

15. Baldini PM, Lentini A, Mattioli P, Provenzano B, De Vito P, et al. (2006) Decrease of polyamine levels and enhancement of transglutaminase activity in selective reduction of B16-F10 melanoma cell proliferation induced by atrial natriuretic peptide. Melanoma Res 16: 501-507.

16. Serafino A, Moroni N, Psaila R, Zonfrillo M, Andreola F, et al. (2012) Antiproliferative effect of atrial natriuretic peptide on colorectal cancer cells: Evidence for an Akt-mediated crosstalk between NHE-1 activity and Wnt/beta-catenin signaling. Biochim Biophys Acta 1822: 1004-1018.

17. Bell EN, Tse MY, Frederiksen LJ, Gardhouse A, Pang SC, et al. (2007) Atrial natriuretic peptide attenuates hypoxia induced chemoresistance in prostate cancer cells. J Urol 177: 751-756. 
18. Zhang J, Zhao Z, Zu C, Hu H, Shen $\mathrm{H}$, et al. (2013) Atrial natriuretic peptide modulates the proliferation of human gastric cancer cells via KCNQ1 expression. Oncol Lett 6: 407-414.

19. Kozlowski MR, Kozlowski RE (2020) A novel, small peptide with activity against human pancreatic cancer. Am J Cancer Res 10: 1356-1365.

20. Eichelbaum EJ, Sun Y, Alli AA, Gower WR, Vesely DL (2008) Cardiac and kidney hormones cure up to $86 \%$ of human small-cell lung cancers in mice. Eur J Clin Invest 38: 562-570.

21. Lenz A, Sun Y, Eichelbaum EJ, Skelton WPt, Pi G, et al. (2010) Twiceweekly intravenous treatment of pancreatic cancer with atrial natriuretic peptide and vessel dilator. In Vivo 24: 125-129.

22. Vesely DL, Eichelbaum EJ, Sun Y, Alli AA, Vesely BA, et al. (2007) Elimination of up to $80 \%$ of human pancreatic adenocarcinomas in athymic mice by cardiac hormones. In Vivo 21: 445-451.

23. Nojiri T, Hosoda H, Tokudome T, Miura K, Ishikane S, Otani K, et al. (2015) Atrial natriuretic peptide prevents cancer metastasis through vascular endothelial cells. Proc Natl Acad Sci USA 112: 4086-4091.

24. Euhus DM, Hudd C, LaRegina MC, Johnson FE (1986) Tumor measurement in the nude mouse. J Surg Oncol 31: 229-234.

ISSN: 2574-1241

DOI: 10.26717/BJSTR.2021.36.005798

Michael R Kozlowski. Biomed J Sci \& Tech Res

This work is licensed under Creative

Commons Attribution 4.0 License

Submission Link: https://biomedres.us/submit-manuscript.php
25. Lenz A, Sun Y, Eichelbaum EJ, Skelton WPt, Pi G, et al. (2010) Cardiac hormones eliminate some human squamous lung carcinomas in athymic mice. Eur J Clin Invest 40: 242-249.

26. Long HJ (1994) Paclitaxel (Taxol): a novel anticancer chemotherapeutic drug. Mayo Clin Proc 69: 341-345.

27. Temel JS, Greer JA, Muzikansky A, Gallagher ER, Admane S, et al. (2010) Early palliative care for patients with metastatic non-small-cell lung cancer. N Engl J Med 363: 733-742.

28. Ferrell BR, Temel JS, Temin S, Alesi ER, Balboni TA, et al. (2017) Integration of Palliative Care into Standard Oncology Care: American Society of Clinical Oncology Clinical Practice Guideline Update. J Clin Oncol 35: 96-112.

29. Reni M, Cereda S, Milella M, Novarino A, Passardi A, et al. (2013) Maintenance sunitinib or observation in metastatic pancreatic adenocarcinoma: a phase II randomized trial. Eur J Cancer 49: 36093615 .

30. Bu L, Baba H, Yoshida N, Miyake K, Yasuda T, et al. (2019) Biological heterogeneity and versatility of cancer-associated fibroblasts in the tumor microenvironment. Oncogene 38: 4887-4901.

$\begin{array}{ll}\text { BIOMEDICAL } & \text { Assets of Publishing with us } \\ \text { RESEARCHES } & \text { - Global archiving of articles } \\ \text { - Immediate, unrestricted online access }\end{array}$

